\title{
Emerging Trends in Nanoparticle Synthesis Using Plant Extracts for Biomedical Applications
}

\author{
Protima Rauwel* and Erwan Rauwel \\ Tallinn University of Technology, Estonia
}

Submission: February 13, 2017; Published: March 09, 2017

*Corresponding author: Protima Rauwel, Tallinn University of Technology, Tel: +372 6204806; Fax: +372 6204801;

Email: protima.rauwel@ttu.ee; erwan.rauwel@ttu.ee

\begin{abstract}
Nanoparticles are of growing importance in biomedical applications and work as anti-bacterial, anti-fungal and anti-cancer agents. Conventional synthesis methods of metal or metal oxide nanoparticles in general, involve toxic reagents which produce harmful by-products and are consequently, hazardous to the environment. For these reasons, Green Chemistry was developed in the 90 s where12 principles towards a sustainable chemistry, were established. Eco-friendly methods of synthesis comprising of plant extracts are now gaining popularity due to the abundance of raw materials and the production of non-toxic by-products benign to the environment. Moreover, the nanoparticles produced by plant-extract-based-green-synthesis methods are cost-effective. The method itself is simple to implement, thereby making it viable for all countries over the world. In addition, nanoparticles produced by green synthesis methods display synergetic properties where both the nanoparticles as well as the natural active molecule of the plant, influence the biocidal properties. Active efforts to control the size and the shape of the nanoparticles are being carried out and new synthesis procedure are regularly being developed. Plant-extractbased-synthesis-routes represent an eco-friendly alternative showing promise in bio-medical applications and in resolving to some extent the negative effects of synthetic drugs.
\end{abstract}

Keywords: Green synthesis; Plant extract; Nanoparticles; Cancer

\section{Introduction}

The last decade has ascertained an emerging trend in plantextract mediated nanoparticle synthesis, notably for noble metal nanoparticles viz., silver or gold. Nanoparticles have been a major center of attraction for diverse scientific investigations for over 20 years, with multiple methods being developed for their synthesis. Nevertheless, the popularity of the bottom-up synthesis approach prevails, even though it implicates chemical reactions engaging toxic or polluting chemicals and producing harmful waste products. Nanomaterials are acknowledged in resolving numerous impending technological challenges in various areas namely, nanomedicine, water purification, soil remediation, catalysis, energy harvesting among a few. Moreover, our daily augmented needs involving nanomaterials will conversely engender an increase in toxic waste production and imperil the environment and human society as a whole. A rising awareness of such possible environmental problems motivated the development of green chemistry in 1990s comprising 12 principles addressing the use and production of hazardous substances in the interest of making chemistry benign by design [1]. These principles emphasize upon the prevention of waste, the minimization of energy during synthesis and the use of renewable raw materials, among others. More particularly, it strives to establish a basis for sustainable chemistry.

The undeniable need for a reliable and an environmentally clean and safe nanoparticle synthesis route has served as a precursor for the development of plant-extract-based-greensynthesis which perfectly meets the required criteria stated in the 12 principles. Furthermore, it makes use of renewable feed stocks like plants, thereby making biosynthesis of metal nanoparticles cost-effective compared to conventional methods. In addition to eco-friendliness, plant-extract-based-synthesis combines the intrinsic properties of plant extracts with the biocidal properties of for example silver nanoparticles via a synergetic effect [2]. Consequently, plant-extract-basedgreen-synthesis methods have developed into a unique branch of investigation with limitless possibilities considering the countless number of plants available across the world.

Plant-extract-based-green-synthesis methods have not only been developed for metal oxide nanoparticles like, $\mathrm{TiO}_{2}, \mathrm{CuO}$ and 
ZnO, but also for metal nanoparticles and more particularly for metallic silver nanoparticles. In fact, colloidal silver has been recognized as an efficient antibacterial agent since Antiquity. Despite a lack of understanding of its biocidal properties, silver nevertheless remains among the most attractive nanomaterials for biomedical applications and further motivates new syntheses routes based on plant extracts. Plant extracts tend to contain numerous organic molecules, each of a different nature like carbohydrates with $s$ at the end, enzymes, aminoacids, proteins, vitamins, alkaloids, etc. that are either potential reducing or stabilizing agents. These molecules are capable of reducing silver ion $\mathrm{Ag}+$ ) from the precursor, leading to the formation of metallic silver (Ag0) and finally agglomerating into nanoparticles. The active molecule that leads to the reduction of $\mathrm{Ag}+$ ions to Ag0, varies with the plant extract employed. During the synthesis process, the active molecule tends to also function as a capping agent, whereupon it functionalizes the surface of the nanoparticles. However, plant extract precursors cannot be considered as pure or uncontaminated as they are generally composed of other organic molecules in addition to the active molecules interacting with the silver precursor.

Plant-extract-based-green-synthesis has a twofold benefit. Firstly, it is an environmentally friendly method as it avoids environmental pollution through reduction or non-production of hazardous by-products and toxic waste. Secondly, it also combines the natural biocidal properties of silver nanoparticles with other specific properties derived from the plant extracts. It is well known that different plant extracts exhibit antiinflammatory, antibacterial, antifungal, antioxidant and even anti-cancer properties. Therefore, sustainable plant extract based green synthesis for metal nanoparticles enables the possibility of combining both metal nanoparticles and plant extract properties via a synergetic effect without the production of toxic waste and employing renewable resources. This synergy opens avenues for these naturally functionalized nanoparticles in anti-bacterial, anti-fungal or anti-cancer treatments, as an answer to the long lasting negative effects of synthetically derived drugs

A peek into the recent reports published in the field validates that the synthesis of nanoparticles using plant extracts, has become an independent branch of Green Chemistry. However, there are still many challenges to overcome with importance given to size and shape control of the nanoparticles and at the same time, avoid the production of silver chloride nanoparticles [3]. A recent review highlighted that in most cases, green synthesis methods produce silver nanoparticles with a broad size distribution and more control is needed to obtain sharper size and shape distributions [4]. The chief reason, as mentioned before, is the purity of the plant extract precursor containing many different organic molecules other than the active one, which interact with the silver precursor. One has also observed fluctuations in the properties of the nanomaterials produced using extracts from the same plants mainly due to differences in extraction methods and the locality where the plants were collected. However, some plant or fruit extract based syntheses have shown sharp size of nanoparticles in certain cases where an accurate control of the synthesis conditions was possible. Therefore, the combination of plant extract properties in the synthesis of metal nanoparticles opens new approaches of investigation for cancer treatment.

\section{Conclusion}

Recent investigations have demonstrated the efficacy of this synergetic effect for cancer treatment. For ex., plant extract of Artemisia marschalliana [5], exhibits anti-oxidant, anti-cancer and anti-microbial properties simultaneously. The main advantage with this synthesis method is that there are no additional reaction steps for the functionalization of the nanoparticles and the method of synthesis is itself rather simple requiring minimum experimental equipment. Nevertheless, increasing the functionality of the green synthesized nanoparticles by adding several other active molecules to the already functionalized nanoparticles has already been demonstrated [5]. The simplicity of the synthesis also provides a plausible explanation for the increasing number of reports in the scientific literature originating from countries that do not possess the same resources as USA, Japan or the European countries. During the next years, we expect to see a leap in this new branch of Green Chemistry as the possibilities appear to be boundless considering the various plant species across the globe, each concealing potent active molecules with distinct biocidal properties. In addition to the cost-effectiveness, the criteria for environmental safety by curbing toxic by-products are achievable via plant-extract-based-green-synthesis. All in all, plant derived nanoparticles are most likely to set the tone for the next generation of bio-medical applications.

\section{Acknowledgment}

The authors wish to acknowledge the Estonian Research Council (Grant PUT431), the European Regional Development Fund project TK134 (TAR16019) for financial support of this work.

\section{References}

1. Paul Anastas, John Warner (1998) Green Chemistry: Theory and Practice (Oxford University Press).

2. Siavash Iravani (2011) Green synthesis of metal nanoparticles using plants. Green Cheml 10(13): 2638-2650.

3. Durán N, Nakazato G, Seabra A B (2016) Antimicrobial activity of biogenic silver nanoparticles, and silver chloride nanoparticles: an overview and comments. Appl Microbiol Biotechnol 100: 6555-6570.

4. Protima Rauwel, Siim Küüna, Stanislav Ferdov, Erwan Rauwel (2015) A Review on the green synthesis of silver nanoparticles and their morphologies studied via TEM. Advances in Materials Science and Engineering pp 1-9.

5. Salehi S, Shandiz SA, Ghanbar F, Darvish MR, Ardestani MS, et al. (2016) Phytosynthesis of silver nanoparticles using Artemisia marschalliana 
Sprengel aerial part extract and assessment of their antioxidant, anticancer and antibacterial properties. Int J Nanomedicine 11: 18351846.

This work is licensed under Creative Commons Attribution 4.0 License

DOI: 10.19080/GJN.2017.01.555562

Your next submission with JuniperPublishers will reach you the below assets

- Quality Editorial service

- Swift Peer Review

- Reprints availability

- E-prints Service

- Manuscript Podcast for convenient understanding

- Global attainment for your research

- Manuscript accessibility in different formats ( Pdf, E-pub, Full Text, Audio)

- Unceasing customer service

Track the below URL for one-step submission https://juniperpublishers.com/submit-manuscript.php 